\title{
Identification and location of ship pipeline leakage based on VMD
}

\author{
Jiechang Wu ${ }^{1}$, Yannan $\mathrm{Li}^{2}$, Shuyong Liu ${ }^{3}$, Xuejiao Feng ${ }^{4}$ \\ $1,2,3$ Naval University of Engineering, Wuhan, China \\ ${ }^{4}$ Hubei Communications Technical College, Wuhan, China \\ ${ }^{1}$ Corresponding author \\ E-mail: ${ }^{1}$ Wujiechang1234@163.com, ${ }^{2} 634053306 @ q q . c o m,{ }^{3}$ lsydh@sina.com, ${ }^{42419463841 @ q q . c o m}$
}

Received 20 September 2019; accepted 1 October 2019

DOI https://doi.org/10.21595/vp.2019.21047

Check for updates

Copyright $(\mathcal{C} 2019$ Jiechang $W u$, et al. This is an open access article distributed under the Creative Commons Attribution License, which permits unrestricted use, distribution, and reproduction in any medium, provided the original work is properly cited.

\begin{abstract}
Pipeline plays an important role in various systems of the ship. However, due to the harsh environment, leakage often occurs in ship pipeline. This paper proposes a method to identity and locate the pipeline leakage. Using the variational mode decomposition (VMD) algorithm, the vibration signal is decomposed into band-limited intrinsic mode functions (BIMFs). The effective BIMFs are then selected by the correlation coefficient. Center frequency and energy value of the effective BIMFs are extracted as feature vector. Radial Basis Function (RBF) neural network is then used as a tool to identify and locate the leakage. The proposed method is finally verified by experiments.
\end{abstract}

Keywords: pipeline leakage, VMD, BIMF, center frequency, RBF neural network.

\section{Introduction}

Pipeline is used to transport liquid or gas and is an important part of various systems in the ship. Ship pipeline leakage occurs frequently due to the harsh working environment and insufficient maintenance. According to the statistical analysis from the former Soviet Union experts, the number of pipeline leakage is only less than the number of the main engine malfunction in the ship [1]. The pipeline leakage are becoming the second most challenging issue in the ship. Even in U.S. Navy, high failure rate caused by corrosion still exists in seawater pipelines and related machinery. Cabin water leakage, fuel leakage and other problems caused by pipeline leakage will expose the equipment and people in the ship to danger. However, there are few methods for pipeline leakage detection in ship. Therefore, it is of great significance to study the pipeline leakage detection technology.

When the pipeline leaks, the pressured flow will result in turbulence, cavitation and friction between the fluid and the pipe wall which will drive the pipeline vibration [2]. The vibration signal travels far and is easy to collect, so the leak detection based on the vibration signal has become an important method for pipeline leak detection.

A large number of studies have been conducted regarding the methods of detecting and locating pipeline leaks based on vibration signals. Wan et al, continuously monitored the leakage signal of the furnace pipe. They used self-adaptive filter to eliminate the noise, and then recognized the leakage by the spectrum analysis of the leakage signal [3]. Li Guanghai used the fast Fourier transform to separate the useful frequency bands of the leakage signal, and then identified leakage based on the power exponential relationship between the power spectral density and the frequency of the useful frequency bands [4]. Majid obtained pipeline leakage signals by experimental method, then extracted the characteristics of leakage signals through wavelet analysis, and used these characteristics to distinguish other signals such as leakage, background noise and pipeline natural vibration [5]. Gu Xiaohong divided the signal into different frequency bands with the method of wavelet packet decomposition. Then, they extracted the energy characteristics and selected the useful frequency bands for the cross-correlation analysis, which reduced the noise interference and improved the positioning accuracy [6]. 


\section{The proposed method}

Leakage signal is a non-stationary random signal, which contains a large amount of information unrelated to the leakage, and must be analyzed to extract the leakage characteristics. Variational mode decomposition (VMD) was proposed by Dragomiretskiy et al. in 2013 [7]. As a kind of non-recursive and self-adaptive signal-processing algorithm, VMD can effectively decompose a multi-component signal into several bandwidth-limited intrinsic mode functions (BIMFs). It is a very efficient method for processing nonlinear and non-stationary signals.

The specific leak identification and detection process in this paper is shown in Fig. 1.

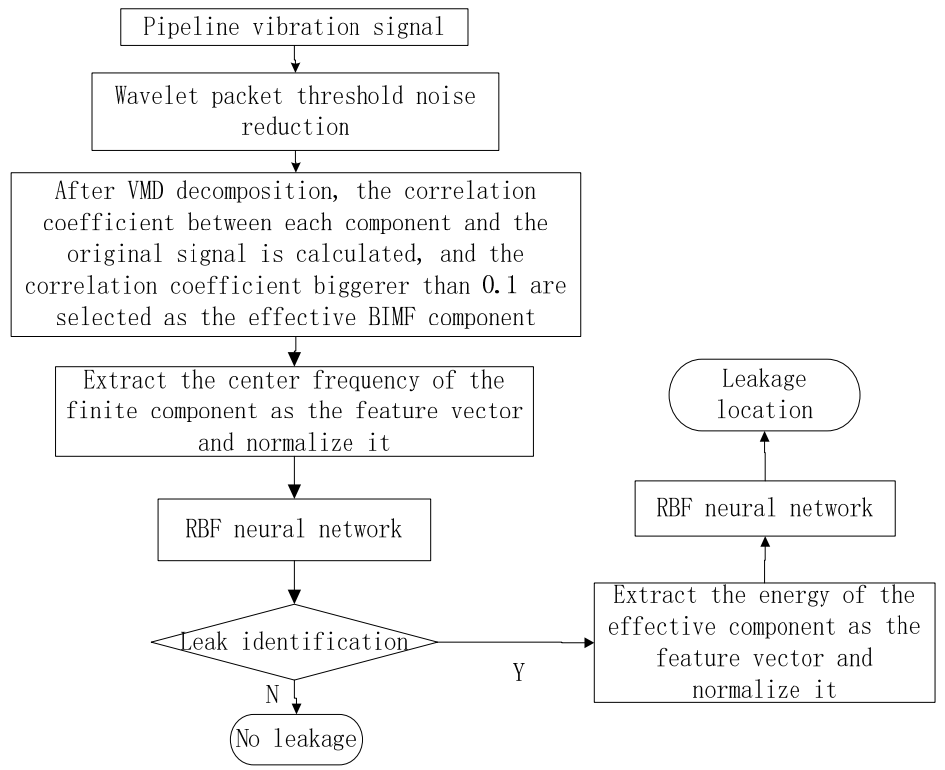

Fig. 1. The flow chart of pipeline leak identification and detection

Due to the influence of environmental noise, direct VMD of the signal will lead to confusions or a large number of noise components in the analysis. Therefore, wavelet packet threshold noise reduction is conducted before signal decomposition in this paper to improve the signal-to-noise ratio. Among the various BIMFs, some are closely related to the leakage signal and are effective components, and the remaining components are not related to leakage or contain a lot of noise. In order to reasonably select the effective BIMFs, this paper introduces the concept of Pearson correlation coefficient. The correlation coefficient of two random discrete signals $x$ and $y$ is defined as [8]:

$\rho_{x y}=\frac{\sum_{i=1}^{n}\left[\left(x_{i}-\bar{x}\right)\left(y_{i}-\bar{y}\right)\right]}{\left[\sum_{i=1}^{n}\left(x_{i}-\bar{x}\right)^{2} \sum_{i=1}^{n}\left(y_{i}-\bar{y}\right)^{2}\right]^{1 / 2}}$.

In the equation, $n$ is the number of discrete points of the signal, $\bar{x}$ and $\bar{y}$ are the mean values of two signal sequences respectively. The absolute value of $\rho_{x y}$ is between 0 and 1 . The closer to 1 , the stronger the correlation between the two signals, and the closer to 0 , the weaker the correlation between the two sets of signals. The correlation coefficient between all BIMFs and the original signal were calculated, and the components whose correlation coefficient was bigger than the set threshold (generally 0.1) [9] were selected as the effective BIMFs.

The research of Osama Hunaidi shows that the frequency distribution of the leakage signal is not random. The distribution falls in a certain frequency band [10]. Therefore, in this paper, the center frequency of the effective BIMFs are used as the feature vector to identify the leakage. 
When the position of the leak point changes, the frequency distribution will not change; however, the energy value of each frequency band of the vibration signal will change, so the energy of the effective BIMFs is used as the feature vector to locate the leak point.

For the extracted feature vectors, this paper uses radial basis function (RBF) neural network as a tool to train and test the samples. This neural network was proposed by Moody et al. in 1989 [11] and it is a neural network with no forward feedback and local approximation. The mapping from input layer to output layer of RBF neural network is non-linear, while the mapping from hidden layer space to output space is linear, so the learning speed is fast and local minimum can be avoided [12]. Due to its strong generalization ability, it is very suitable for online monitoring.

\section{Test verification}

In order to verify the proposed method, a pipeline leakage test platform was built in the laboratory to simulate the ship pipeline. In order to simulate the noise on the ship, the pump is used to supply water to the test pipeline, and the influence of the noise of the pump is added to the collected signal. At the same time, in order to simulate the narrow environment on the ship, the test platform is placed in a small space. The pipeline system is shown in Fig. 2. Section AB is the main test pipeline. Four (4) leakage holes with a diameter of $4 \mathrm{~mm}$ were drilled in the pipeline to simulate the leakage. The layout of the leakage points is shown in Fig. 3. Under the same working condition, 10 sets of vibration signal data were collected for 5 different scenarios including no leakage and leakage occurred at each of the holes. The sampling frequency was $10000 \mathrm{~Hz}$ and the sampling time was $1 \mathrm{~s}$. Total 50 sets of data samples were collected for the training and verification of neural network. The method discussed above is used to analyze the data samples and to extract the feature vectors of effective BIMFs. The center frequency distribution of no leakage signal is shown in Fig. 4. The center frequency distribution of leakage signal is shown in Fig. 5.

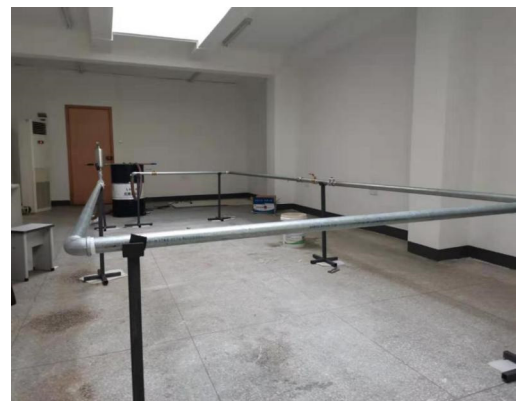

Fig. 2. Experimental device



Fig. 3. Distribution diagram of leakage points

Figs. 4 and 5 shows that when the pipeline leaks, the center frequency of the effective BIMFs of the vibration signal will mainly distribute in $25-50 \mathrm{~Hz}, 500-750 \mathrm{~Hz}, 850-1100 \mathrm{~Hz}$ and $1200-1400 \mathrm{~Hz}$. While the center frequency of BIMF components of the non-leakage pipeline vibration signal are lower than that of the leakage signal. The center frequency eigenvectors of 40 groups of samples are selected to train the neural network. The output layer of the neural network is 0 or 1,0 means there is no leakage, and 1 means there is leakage in the pipeline. The remaining 10 groups of sample data are used for verification. The verification results are shown in the Table 1 . 


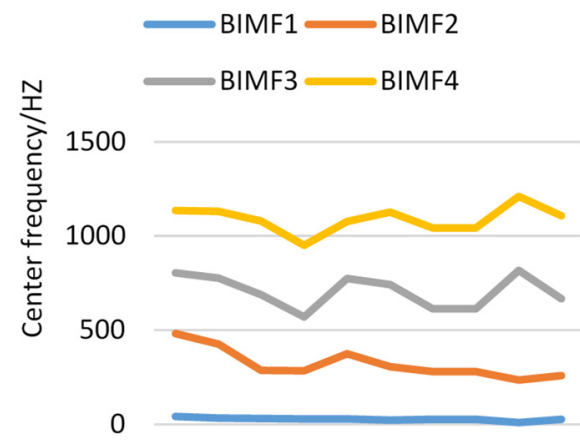

Fig. 4. No leakage signal


Fig. 5. Leakage signal

Table 1. Neural network identification results

\begin{tabular}{|c|c|c|c|c|}
\hline Sample number & Pipeline state & Desired output & Actual output & Identification results \\
\hline 1 & Leakage & 1 & 1.0297 & Leakage \\
\hline 2 & Leakage & 1 & 1.0080 & Leakage \\
\hline 3 & Leakage & 1 & 0.9591 & Leakage \\
\hline 4 & Leakage & 1 & 1.0672 & Leakage \\
\hline 5 & Leakage & 1 & 0.8107 & Leakage \\
\hline 6 & Leakage & 1 & 1.0023 & Leakage \\
\hline 7 & Leakage & 1 & 0.9422 & Leakage \\
\hline 8 & Leakage & 1 & 1.0543 & Leakage \\
\hline 9 & No leakage & 0 & -0.4908 & Unable to identify \\
\hline 10 & No leakage & 0 & -0.0451 & No leakage \\
\hline
\end{tabular}

The energy rate of the effective BIMFs of the vibration signal of the leakage pipeline is shown in the Fig. 6.

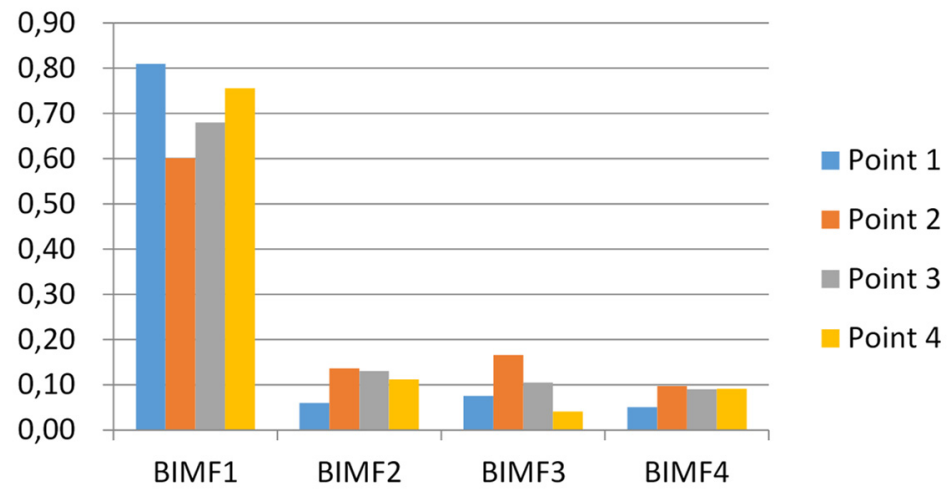

Fig. 6. The energy rate of leakage signal

Fig. 6 shows that the energy distribution is different at different leak locations. The energy rate eigenvectors of 32 leakage signal samples are selected to train the neural network. The output of the network are $(0,0),(0,1),(1,0)$ and $(1,1)$, respectively indicating points $1,2,3$ and point 4 leakage occurs. The remaining 8 sets of samples are used in the trained neural network to locate the leak point. The results are shown in the Table 2 .

Table 1 and Table 2 indicate that the results of the neural network output are not 0 or 1 as desired, but the actual outputs are close to the expected outputs. Except for the two results that unable to be located, all the others can be identified and positioned correctly. The feasibility and high accuracy of the proposed method are verified. 
Table 2. Neural network location results

\begin{tabular}{|c|c|c|c|c|}
\hline Sample number & Leak location & Desired output & Actual output & Location results \\
\hline 1 & 1 & $(0,0)$ & $(0.1025,0.0076)$ & Point 1 \\
\hline 2 & 1 & $(0,0)$ & $(-0.1379,-0.6187)$ & Unable to locate \\
\hline 3 & 2 & $(0,1)$ & $(-0.1141,0.9023)$ & Point 2 \\
\hline 4 & 2 & $(0,1)$ & $(-0.0282,0.8579)$ & Point 2 \\
\hline 5 & 3 & $(1,0)$ & $(0.8103,0.1420)$ & Point 3 \\
\hline 6 & 3 & $(1,0)$ & $(0.9106,0.0198)$ & Point 3 \\
\hline 7 & 4 & $(1,1)$ & $(1.1474,0.9920)$ & Point 4 \\
\hline 8 & 4 & $(1,1)$ & $(0.9520,1.0278)$ & Point 4 \\
\hline
\end{tabular}

\section{Conclusions}

This paper proposes a signal time-frequency analysis method, a combination of VMD and RBF neural network, to identify and locate leakage in ship pipelines. The center frequency and energy of effective BIMFs are used to identify and locate leakage. The experiment results proved that the proposed method has a relatively high accuracy.

The pressure difference between the internal and external vessels of the ship is large, and the vibration signal is obvious due to the leakage. The method combines the wavelet packet threshold denoising and the VMD to have a strong noise reduction capability, so it has high application value on the ship. However, for small leaks, the vibration signal is not obvious. The next step is to study the feature extraction of weak signals in strong background noise.

\section{Acknowledgements}

The research is support by the provincial foundation of China (2017CFB672) and University Scientific foundation (425517 K143).

\section{References}

[1] Zhou Yongfeng, Wang Hongren Research progress on environmental corrosion of Marine seawater piping. Materials Development and Application, Vol. 3, 2009, p. 16-20+23.

[2] Zhang Minzi Study on Location of Water Pipeline Leakage in the Presence of $90^{\circ}$ Bend. Chongqing University, 2017.

[3] Qin Wan, Koch Daniel Multichannel spectral analysis for tube leak detection. Proceedings of Southeastcon, 1993.

[4] Li Guanghai, Liu Shifeng, Geng Rongsheng, et al. A new method for feature identification of acoustic emission sources. Nondestructive Testing, Vol. 24, Issue 12, 2009, p. 534-538.

[5] Ahadi M., Bakhtiar M. S. Leak detection in water-filled plastic pipes through the application of tuned wavelet transforms to acoustic emission signals. Applied Acoustics, Vol. 71, Issue 7, 2010, p. 634-639.

[6] Gu Xiaohong, Zhang Guangxin, Hou dibo, et al. Determination of water pipe leakage location by combining wavelet packet decomposition and energy feature extraction. Engineering Science and Technology, Vol. 37, Issue 6, 2010, p. 145-149.

[7] Dragomiretskiy K., Zosso D. Variational mode decomposition. IEEE Transactions on Signal Processing, Vol. 62, Issue 3, 2010, p. 531-544. 